\title{
LOCAL TRIPLE DERIVATIONS FROM C* -ALGEBRAS INTO THEIR ITERATED DUALS
}

\author{
MOHSEN NIAZI AND MOHAMMAD REZA MIRI
}

\begin{abstract}
We show that every local triple derivation from a $\mathrm{C}^{*}$-algebra into any of its iterated duals is a triple derivation. This result partially solves a problem posed by M. Burgos et al. in [Bull. London Math. Soc. 46 (4), 709-724 (2014)].
\end{abstract}

\section{Introduction}

In [11], M. Mackey introduced the notion of local triple derivations on Jordan triples. A linear mapping $T$ on a Jordan triple $E$ is called a local triple derivation if for each $a \in E$ there exists a triple derivation $D_{a}$ on $E$, depending on $a$, such that $T(a)=D_{a}(a)$. Mackey proved that every continuous local triple derivation on a JBW* ${ }^{*}$-triple is a triple derivation (cf. [11, Theorem 5.11]). This is a counterpart result to the Kadison's theorem in the category of binary (associative) algebras which shows that every continuous local derivation on a von Neumann algebra is a derivation [7].

In [2], M. Burgos et al. improved the Mackey's result for $\mathrm{C}^{*}$-algebras. They considered a $\mathrm{C}^{*}$-algebra $A$ as a Jordan triple with the following triple product:

$$
[a, b, c]=\frac{1}{2}\left(a b^{*} c+c b^{*} a\right), \quad(a, b, c \in A) .
$$

This result was a partial positive answer to the question: "Is a local triple derivation on a JB* triple a triple derivation?” posed by M. Mackey in [11, Conjecture 6.2]. This line of researches had been continued and finally provided a complete positive answer to the just quoted conjecture. In [3, Theorem 2.4] M. Burgos et al. proved that every bounded local triple derivation on a JB*-triple is a triple derivation. After solving this problem they posed in [3] another conjecture: “Is a local triple derivation from a JB*-triple into its dual a triple derivation?", where the dual of a JB* -triple is considered as a ternary module.

Received June 6, 2016, accepted December 7, 2017.

2010 Mathematics Subject Classification. Primary 47B47, 46L57; Secondary 17C65, 46L05, 46L08.

Key words and phrases. triple derivation, local triple derivation, derivation, generalised derivation, unital $\mathrm{C}^{*}$-algebra.

Corresponding author: Mohsen Niazi. 
The study of module-valued triple derivations on Jordan triples initiated by B. Russo and A. M. Peralta in [14]. They proposed a ternary module structure on Jordan triples and showed that by defining appropriate ternary module actions, the dual of a Jordan triple can be endowed with a ternary module structure. While the proposed structure make it possible to consider the dual of a Jordan triple as a ternary module, it fails to induce a ternary module structure on the iterated duals of a Jordan triple. To remedy this pathology the authors of this paper proposed another type of ternary module structure in [13], which combined with the previous one exhibit a complete picture of the module structures in the category of Jordan triples (cf. Definition 2.1 and Theorem 2.3 in [13]).

In this paper we provide a partial positive answer to the Problem 2.7 in [3]. We prove in Theorem 3.9 that every continuous local triple derivation from a $\mathrm{C}^{*}$-algebra into any of its iterated duals, which are considered as ternary modules, is a triple derivation.

To provide a reasonable discussion of the ternary module actions we devote the next section to review necessary definitions and results on Jordan triples and ternary modules.

\section{Jordan triples and ternary modules}

In this section we recall definitions and some basic facts about Jordan triples, ternary modules and construct ternary module structures on the iterated duals of a Jordan Banach triple.

\subsection{Jordan triples}

Let $E$ be a complex vector space. A triple product on $E$ is a mapping

$$
\pi: E \times E \times E \rightarrow E, \pi(x, y, z)=[x, y, z]
$$

which is bilinear and symmetric in the outer variables and conjugate linear in the middle one satisfying the so-called "Jordan Identity":

$$
[a, b,[c, d, e]]=[[a, b, c], d, e]-[c,[b, a, d], e]+[c, d,[a, b, e]],
$$

for all $a, b, c, d, e$ in $E$. The pair of $(E, \pi)$ is called a Jordan triple. When $E$ is a Banach space and the triple product of $E$ is continuous, we say that $E$ is a Jordan Banach triple.

A $J B^{*}$-triple is a Jordan Banach triple $E$ satisfying the following axioms:

(1) For any $a$ in $E$ the mapping $x \mapsto[a, a, x]$ is a hermitian operator on $E$ with non-negative spectrum;

(2) $\|[a, a, a]\|=\|a\|^{3}$ for all $a$ in $A$. 
To provide an extension of the triple product of a Jordan triple on its bidual, firstly we make the following definitions. In the most general case, let $X, Y, Z$ and $W$ be Banach spaces and $f: X \times Y \times Z \rightarrow W$ be a continuous map which is linear or conjugate linear in each of its variables. Define the transpose $f^{*}$ of $f$ by

$$
f^{*}: W^{*} \times X \times Y \rightarrow Z^{*},\left\langle f^{*}\left(w^{*}, x, y\right), z\right\rangle=\left\langle w^{*}, f(x, y, z)\right\rangle
$$

whenever $f$ is linear in the third variable and define the conjugate transpose $f^{\sharp}$ of $f$ by

$$
f^{\sharp}: W^{*} \times X \times Y \rightarrow Z^{*},\left\langle f^{*}\left(w^{*}, x, y\right), z\right\rangle=\overline{\left\langle w^{*}, f(x, y, z)\right\rangle}
$$

whenever $f$ is conjugate linear in the third variable. It is easy to see that both of the maps $f^{*}$ and $f^{\sharp}$ are $w^{*}-w^{*}$-continuous in the first variable.

Now let $E$ be a Jordan triple with triple product $\pi$. Since $\pi$ is linear in the third variable, we can apply definition (3) and obtain the following transpose of $\pi$ :

$$
\pi^{*}: E^{*} \times E \times E \rightarrow E^{*}
$$

It is easy to see that $\pi^{*}$ is conjugate linear in the third variable. So we can apply definition (4) and obtain the following conjugate transpose of $\pi^{*}$ :

$$
\pi^{* \sharp}:=\left(\pi^{*}\right)^{\sharp}: E^{* *} \times E^{*} \times E \rightarrow E^{*} .
$$

An easy verification shows that $\pi^{* \sharp}$ is conjugate linear in the third variable. Another application of definition (4) results the following conjugate transpose of $\pi^{* \sharp}$ :

$$
\pi^{* \sharp \sharp}:=\left(\pi^{* \sharp}\right)^{\sharp}: E^{* *} \times E^{* *} \times E^{*} \rightarrow E^{*},
$$

which is linear in the third variable. Finally we apply definition (3) and obtain the following transpose of $\pi^{* \# \text { : }}$

$$
\pi^{* \# \# *}:=\left(\pi^{* \# \#}\right)^{*}: E^{* *} \times E^{* *} \times E^{* *} \rightarrow E^{* *} .
$$

The following proposition is an easy observation.

Proposition 2.1. Let $E$ be a Jordan triple with triple product $\pi$. Then $\pi^{* \# * *}$ is an extension of $\pi$ to the bidual space $E^{* *}$ and the following assignments:

$$
\begin{aligned}
& x^{* *} \mapsto \pi^{* \sharp \# *}\left(x^{* *}, y^{* *}, z^{* *}\right), \quad\left(y^{* *}, z^{* *} \in E^{* *}\right), \\
& y^{* *} \mapsto \pi^{* \sharp \# *}\left(x, y^{* *}, z^{* *}\right), \quad\left(x \in E, z^{* *} \in E^{* *}\right), \\
& z^{* *} \mapsto \pi^{* \sharp \# *}\left(x, y, z^{* *}\right), \quad(x, y \in E)
\end{aligned}
$$

are $w^{*}-w^{*}$-continuous maps. 
Let $E$ be a JB*-triple with triple product $\pi$. Theorem 4.5 in [8] shows that $\pi^{* \sharp \# *}$ is a triple product on $E^{* *}$ which make it a JB* -triple (see also [4, Corollary 11]). We see therefore that $E^{(2 n)}$ is a JB* -triple for every $n \in \mathbb{N}$. For simplicity, we use the following notation:

$$
\pi^{[1]}=\pi^{* \sharp \# *}, \pi^{[n+1]}=\pi^{[n] * \# *},(n \in \mathbb{N}) .
$$

\subsection{Ternary modules}

In [14] A.M. Peralta and B. Russo introduced the notion of ternary modules over Jordan triples. Trying to endow the dual of a ternary module with ternary module structure, in [13] the authors of this paper improved the previous notion of ternary modules over Jordan triples by introducing a new type of ternary modules and called it ternary module of type (II). We recall both of them in the following:

Definition 2.2. Let $E$ be a Jordan triple and $X$ be a complex vector space. Consider the following mappings and axioms:

$$
\begin{aligned}
& \pi_{1}: X \times E \times E \rightarrow X, \pi_{1}(x, a, b)=[x, a, b]_{1}, \\
& \pi_{2}: E \times X \times E \rightarrow X, \pi_{2}(a, x, b)=[a, x, b]_{2}, \\
& \pi_{3}: E \times E \times X \rightarrow X, \pi_{3}(a, b, x)=[a, b, x]_{3} .
\end{aligned}
$$

(1) $\pi_{1}$ is linear in the first and second variables an conjugate linear in the third variable. $\pi_{2}$ is conjugate linear in each variable. $\pi_{3}$ is conjugate linear in the first variable and linear in the second and third variables.

(1)' Each of the mappings $\pi_{1}, \pi_{2}$ and $\pi_{3}$ is linear in the first and third variables and conjugate linear in the second variable.

(2) $[x, b, a]_{1}=[a, b, x]_{3}$, and $[a, x, b]_{2}=[b, x, a]_{2}$ for every $a, b \in E$ and $x \in X$.

(3) Let $[\cdot, \cdot, \cdot]$ denotes any of the mappings $[\cdot, \cdot, \cdot]_{1},[\cdot, \cdot, \cdot]_{2},[\cdot, \cdot, \cdot]_{3}$ or the triple product of $E$. Then the following identity

$$
[a, b,[c, d, e]]=[[a, b, c], d, e]-[c,[b, a, d], e]+[c, d,[a, b, e]]
$$

holds for every $a, b, c, d, e$ where one of them is in $X$ and the other ones are in $E$.

When the mappings $\pi_{1}, \pi_{2}$ and $\pi_{3}$ satisfy the axioms (1), (2) and (3), $X$ is called a ternary E-module of type $(I)$ and when they satisfy the axioms (1)' ${ }^{\prime}(2)$ and (3), $X$ is called a ternary E-module of type $(I I)$.

Note that axiom (3) of the above definition consists of five identities regarding the position of the module element. Henceforth, we write the expression "ternary $E$-module”, without 
declaring the type, whenever the type is clear from the context or a statement is true for both types.

When $E$ is a Jordan Banach triple, $X$ is a Banach space and the module actions $\pi_{1}, \pi_{2}$ and $\pi_{3}$ are continuous we say that $X$ is a Banach ternary $E$-module.

To simplify notations, hereafter, the module actions $[\cdot, \cdot, \cdot]_{1},[\cdot, \cdot, \cdot]_{2},[\cdot, \cdot, \cdot]_{3}$ and the triple product of $E$ will be denoted by $[\cdot, \cdot, \cdot]$ and its meaning will be clear from the context.

To provide clear definitions of module actions on the dual of a ternary module, we introduce the following three maps. Let $X, Y, Z$ and $W$ be Banach spaces and $f: X \times Y \times Z \rightarrow W$ be a continuous map which is linear or conjugate linear in each of its variables. Let

$$
f^{1}: W^{*} \times X \times Y \rightarrow Z^{*},\left\langle f^{1}\left(w^{*}, x, y\right), z\right\rangle=\left\langle w^{*}, f(x, y, z)\right\rangle,
$$

whenever $f$ is linear in the third variable,

$$
f^{2}: X \times W^{*} \times Z \rightarrow Y^{*},\left\langle f^{2}\left(x, w^{*}, z\right), y\right\rangle=\overline{\left\langle w^{*}, f(x, y, z)\right\rangle},
$$

whenever $f$ is conjugate linear in the second variable, and

$$
f^{3}: Y \times Z \times W^{*} \rightarrow X^{*},\left\langle f^{3}\left(y, z, w^{*}\right), x\right\rangle=\left\langle w^{*}, f(x, y, z)\right\rangle,
$$

whenever $f$ is linear in the first variable. Note that $f^{1}=f^{*}$.

Let $E$ be a Jordan triple and $\left(X ; \pi_{1}, \pi_{2}, \pi_{3}\right)$ be a ternary $E$-module. Theorem 2.3 in [13] shows that $\left(X^{*} ; \pi_{3}^{1}, \pi_{2}^{2}, \pi_{1}^{3}\right)$ is a ternary $E$-module of type (II) whenever $X$ is of type (I) and is a ternary $E$-module of type (I) whenever $X$ is of type (II).

It is easy to see that every Jordan Banach triple $E$ with a triple product $\pi$ is a Banach ternary $E$-module of type (II) when $\pi$ is considered as its module actions, i.e. $\pi_{1}=\pi_{2}=\pi_{3}:=$ $\pi$. The argument in the preceding paragraph shows that $\left(E^{*} ; \pi^{1}, \pi^{2}, \pi^{3}\right)$ is a Banach ternary $E$-module of type (I), $\left(E^{* *} ; \pi^{31}, \pi^{22}, \pi^{13}\right)$ is a Banach ternary $E$-module of type (II), ... etc. This procedure shows that any iterated dual spaces $E^{(n)}$ of $E$ is a Banach ternary $E$-module of type (I) with module actions

$$
\pi^{131 \cdots 1}, \pi^{222 \cdots 2} \text { and } \pi^{313 \cdots 3},
$$

whenever the integer $n$ is odd and $E^{(n)}$ is a Banach ternary $E$-module of type (II) with module actions

$$
\pi^{313 \cdots 1}, \pi^{222 \cdots 2}, \pi^{131 \cdots 3}
$$

whenever the integer $n$ is even.

We restate the following proposition from [13] which provides a clear picture of the module actions of the iterated duals of a Jordan Banach triple $E$. 
Proposition 2.3. Let E be Jordan Banach triple with triple product $\pi$ and $n \in \mathbb{Z}^{+}$. Let we denote the module actions of $E^{(2 n)}$ by $\omega_{n, 1}, \omega_{n, 2}, \omega_{n, 3}$. Then

$$
\omega_{n, 1}=\left.\pi^{[n]}\right|_{E^{(2 n)} \times E \times E}, \omega_{n, 2}=\left.\pi^{[n]}\right|_{E \times E^{(2 n)} \times E}, \omega_{n, 3}=\left.\pi^{[n]}\right|_{E \times E \times E^{(2 n)}} .
$$

Applying this result we obtain module actions of the odd and even iterated duals of a Jordan Banach triple $(E, \pi)$, as the following:

$$
\left(E^{(2 n)}, \pi^{[n]}, \pi^{[n]}, \pi^{[n]}\right) \text { and }\left(E^{(2 n+1)}, \pi^{[n] 1}, \pi^{[n] 2}, \pi^{[n] 3}\right),
$$

where $n \in \mathbb{Z}^{+}$.

Let $A$ be a Banach *-algebra. The identity (1) defines a triple product on $A$. Hence, by the rule described in the preceding paragraphs it turns out that the iterated dual spaces $A^{(n)}$ of $A$ are ternary $A$-modules. Note that the iterated dual spaces $A^{(n)}$ also enjoy binary $A$-bimodule structures by the following recursively defined module actions:

$$
(a \theta)(\varphi)=\theta(\varphi a), \quad(\theta a)(\varphi)=\theta(a \varphi)
$$

where $a \in A, \theta \in A^{(n)}$ and $\varphi \in A^{(n-1)}$. We also define recursively an involution * on $A^{(n)}$ by

$$
\theta^{*}(\varphi)=\overline{\theta\left(\varphi^{*}\right)}, \quad\left(\theta \in A^{(n)}, \varphi \in A^{(n-1)}\right) .
$$

The following proposition determine the relationship between ternary module actions and binary module actions on the iterated dual spaces of a Banach * -algebra. Its proof is an easy verification of the definitions of module actions.

Proposition 2.4. Let $A$ be a Banach ${ }^{*}$-algebra and $n \in \mathbb{N}$. For every $a, b \in A$ and $\theta \in A^{(n)}$, we have

$$
[\theta, a, b]=[b, a, \theta]=\frac{1}{2}\left(\theta a b^{*}+b^{*} a \theta\right), \quad[a, \theta, b]=\frac{1}{2}\left(a^{*} \theta^{*} b^{*}+b^{*} \theta^{*} a^{*}\right),
$$

whenever $n$ is odd, and

$$
[\theta, a, b]=[b, a, \theta]=\frac{1}{2}\left(\theta a^{*} b+b a^{*} \theta\right), \quad[a, \theta, b]=\frac{1}{2}\left(a \theta^{*} b+b \theta^{*} a\right),
$$

whenever $n$ is even.

Regarding two different types of ternary modules it is natural to have two different types of derivations:

Definition 2.5. A triple derivation from a Jordan triple $E$ into a ternary $E$-module of type (I) (resp. (II)) $X$ is a conjugate linear (resp. linear) mapping $D: E \rightarrow X$, satisfying

$$
D[a, b, c]=[D a, b, c]+[a, D b, c]+[a, b, D c],
$$

for every $a, b, c$ in $E$. 
Note the conjugate linearity of a triple derivation when its codomain is a ternary module of type (I) and linearity of the one when its codomain is a ternary module of type (II). This difference will always be realized from the intended type of the module.

Let $E$ be a Jordan triple and $X$ be a ternary $E$-module. Applying the axiom (3) of Definitions 2.2, for every $b \in E$ and $x \in X$, we see that the mapping $\delta(b, x): E \rightarrow X$, defined by

$$
\delta(b, x)(a)=[b, x, a]-[x, b, a], \quad(a \in E)
$$

is a triple derivation. A finite sum of the above derivations (9) is called a inner ternary derivation.

We also recall that a derivation from a binary (associative) algebra $B$ into an $E$-bimodule $X$ is a linear mapping $D: B \rightarrow X$ satisfying the following identity:

$$
D(a b)=D(a) b+a D(b), \quad(a, b \in B) .
$$

It is called a Jordan derivation if for every $a \in B, D\left(a^{2}\right)=D(a) a+a D(a)$ or equivalently

$$
D(a \circ b)=D(a) \circ b+a \circ D(b),(a, b \in B),
$$

where $a \circ b=(a b+b a) / 2$.

If the algebra $B$ is unital, a linear mapping $D: B \rightarrow X$ is called a generalised derivation if it satisfies the following identity:

$$
D(a b)=D(a) b+a D(b)-a D(1) b, \quad(a, b \in B) .
$$

Note that a generalized derivation would be a derivation if it vanishes at the identity of the algebra.

\section{Module-valued local triple derivations}

The results in this section are primarily extensions of the results in [2]. Also the techniques we apply in this development are almost the same as ones in [2], except for the results concerning weak* continuity of the module actions. In general ternary module actions are not necessarily separately $w^{*}$-continuous. In Proposition 3.4 we show that the lack of separately weak* continuity of the ternary module actions on the iterated duals of a $\mathrm{C}^{*}$-algebra is not a real obstacle in our development.

Lemma 3.1. Let $B$ be a $C^{*}$-algebra, $A$ be a commutative closed subalgebra of $B$ and $T: A \rightarrow B^{(n)}$ be a local triple derivation. Then $[a, T(b), c]=0$ for every $a, b, c$ in $A$ with $a^{*} b=b^{*} c=0$. 
Proof. Let $a, b, c$ be elements in $A$ satisfying $a^{*} b=b^{*} c=0$. By assumption there exists a triple derivation $D_{b}: A \rightarrow B^{(n)}$ such that $T(b)=D_{b}(b)$. The identity

$$
[a, T(b), c]=\left[a, D_{b}(b), c\right]=D_{b}[a, b, c]-\left[D_{b}(a), b, c\right]-\left[a, b, D_{b}(c)\right] .
$$

combined with the following identities obtained from Proposition 2.4

and

$$
\begin{aligned}
D_{b}[a, b, c] & =\frac{1}{2} D_{b}\left(a b^{*} c+c b^{*} a\right)=0, \\
{\left[D_{b}(a), b, c\right] } & \left.=\frac{1}{2}\left(D_{b}(a) b^{*} c+c b^{*} D_{(} a\right)\right)=0,
\end{aligned}
$$

$$
\left[a, b, D_{b}(c)\right]=\frac{1}{2}\left(a b^{*} D_{b}(c)+D_{b}(c) b^{*} a\right)=0,
$$

whenever $n$ is even, and

$$
\begin{aligned}
D_{b}[a, b, c] & =\frac{1}{2} D_{b}\left(a b^{*} c+c b^{*} a\right)=0, \\
{\left[D_{b}(a), b, c\right] } & \left.=\frac{1}{2}\left(D_{b}(a) b c^{*}+c^{*} b D_{(} a\right)\right)=0,
\end{aligned}
$$

and

$$
\left[a, b, D_{b}(c)\right]=\frac{1}{2}\left(a^{*} b D_{b}(c)+D_{b}(c) b a^{*}\right)=0,
$$

whenever $\mathrm{n}$ is odd, proves the statement.

Lemma 3.2. Let $A$ be a commutative unital $C^{*}$-algebra. Let $X$ be a Banach space and $S: A \times$ $A \rightarrow X$ be a continuous mapping which is linear in the first variable and conjugate linear in the second variable. If $S(x, y)=0$ for every $x, y \in A$ with $x^{*} y=0$, then

$$
S(x, y)=S\left(1, x^{*} y\right), \quad(x, y \in A) .
$$

Proof. For every $\phi \in X^{*}$, by Theorem 1.10 in [5], there exists $\psi_{1}, \psi_{2} \in A^{*}$, such that

$$
\phi \circ S(x, y)=\psi_{1}\left(y^{*} x\right)+\psi_{2}\left(x y^{*}\right), \quad(x, y \in A) .
$$

Since $A$ is commutative, for every $x, y \in A$, we have

$$
\phi \circ S(x, y)=\left(\psi_{1}+\psi_{2}\right)\left(y^{*} x\right)
$$

From this identity, we also obtain

$$
\phi \circ S\left(1, x^{*} y\right)=\left(\psi_{1}+\psi_{2}\right)\left(y^{*} x\right)
$$

which combined with identity (10) proves that $\phi \circ S(x, y)=\phi \circ S\left(1, x^{*} y\right)$. The desired result follows from Hahn-Banach theorem. 
Proposition 3.3. Let $B$ be a unital $C^{*}$-algebra and $A$ be a commutative closed subalgebra of $B$ containing the identity of $B$. For every bounded local triple derivation $T: A \rightarrow B^{(n)}$, we have the following identity

$$
[x, T(y z), w]=\left[x, T(y), z^{*} w\right]+\left[y^{*} x, T(z), w\right]-\left[y^{*} x, T(1), z^{*} w\right]
$$

where $x, y, z, w \in A$.

Proof. Let $n$ be an odd integer. Let $x, y \in A$, and define

$$
U_{x, y}: A \times A \rightarrow B^{(n)}, U_{x, y}(z, w)=[x, T(y z), w] .
$$

Applying Proposition 2.4, we see that

$$
U_{x, y}(z, w)=\frac{1}{2}\left(x^{*} T(y z)^{*} w^{*}+w^{*} T(y z)^{*} x^{*}\right),
$$

for every $z, w \in A$. Having in mind that by definition $T: A \rightarrow B^{(n)}$ is a conjugate linear mapping in the odd cases of $n$, we deduce from above identity that $U_{x, y}(z, w)$ is linear in $z$ and conjugate linear in $w$. If $x^{*} y=0$ then Lemma 3.1 assures that $U_{x, y}(z, w)=0$, for every $z, w \in A$ with $z^{*} w=0$. Applying Lemma 3.2, we obtain

$$
[x, T(y z), w]=U_{x, y}(z, w)=U_{x, y}\left(1, z^{*} w\right)=\left[x, T(y), z^{*} w\right],
$$

for every $x, y, z, w \in A$ with $x^{*} y=0$.

Let $z, w \in A$, and define

$$
V_{z, w}: A \times A \rightarrow B^{(n)}, V_{z, w}(y, x)=[x, T(y z), w]-\left[x, T(y), z^{*} w\right] .
$$

Applying Proposition 2.4 and considering that $T$ is a conjugate linear mapping, we see that $V_{z, w}(y, x)$ is linear in $y$ and conjugate linear in $x$. The above equation (12) shows that $V_{z, w}(y, x)$ $=0$ for every $y, x \in A$ with $y^{*} x=0$. Hence Lemma 3.2 implies that $V_{z, w}(y, x)=V_{z, w}\left(1, y^{*} x\right)$, for all $x, y \in A$, which concludes the desired identity.

The same argument works for even integers except for slight changes in conjugacy of the variables and involutions.

In the following proposition we extend the identity (11) to the second dual of the corresponding spaces.

Proposition 3.4. Let $B$ be a unital $C^{*}$-algebra and $A$ be a commutative closed subalgebra of $B$ containing the identity of $B$. For every bounded local triple derivation $T: A \rightarrow B^{(n)}$, we have the following identity

$$
\left[x, T^{* *}(y z), w\right]=\left[x, T(y), z^{*} w\right]+\left[x y^{*}, T^{* *}(z), w\right]-\left[x y^{*}, T(1), z^{*} w\right] .
$$

where $y \in A$ and $x, z, w \in A^{* *}$. 
Proof. Let $T: A \rightarrow B^{(n)}$ be a bounded local triple derivation and $T^{* *}: A^{* *} \rightarrow B^{(n+2)}$ be its second adjoint which is a $w^{*}-w^{*}$-continuous mapping. In the sequel we prove the statement in two different case of even and odd for the nonnegative integer $n$.

Let $n=2 k$ be an even integer. Let $y \in A, x, z, w \in A^{* *}$ and $\left\{x_{\alpha}\right\},\left\{z_{\beta}\right\}$ and $\left\{w_{\gamma}\right\}$ be bounded nets in $A, w^{*}$-converging to $x, z$ and $w$, respectively. Rewriting the identity (11) in the notation of the expression (8), we have the following identity

$$
\pi^{[k]}\left(x_{\alpha}, T\left(y z_{\beta}\right), w_{\gamma}\right)=\pi^{[k]}\left(x_{\alpha}, T(y), z_{\beta}^{*} w_{\gamma}\right)+\pi^{[k]}\left(x_{\alpha} y^{*}, T\left(z_{\beta}\right), w_{\gamma}\right)-\pi^{[k]}\left(x_{\alpha} y^{*}, T(1), z_{\beta}^{*} w_{\gamma}\right)
$$

for every $x_{\alpha}, z_{\beta}$ and $w_{\gamma}$. Since the product of the $\mathrm{C}^{*}$-algebra $A^{* *}$ is separately $w^{*}$-continuous and so is its involution, we see that the net $\left\{z_{\beta}^{*} w_{\gamma}\right\}$ is $w^{*}$-convergent to $z_{\beta}^{*} w$ for every $\beta$, the net $\left\{z_{\beta}^{*} w\right\}$ is $w^{*}$-convergent to $z^{*} w$ and the net $\left\{x_{\alpha} y^{*}\right\}$ is $w^{*}$-convergent to $x y^{*}$. Proposition 2.1 implies that by taking limits firstly in $\gamma$, then in $\beta$ and after that in $\alpha$, we obtain

$$
\pi^{[k+2]}\left(x, T^{* *}(y z), w\right)=\pi^{[k+2]}\left(x, T(y), z^{*} w\right)+\pi^{[k+2]}\left(x y^{*}, T(z), w\right)-\pi^{[k+2]}\left(x y^{*}, T(1), z^{*} w\right),
$$

which is the desired identity when we rewrite it in the bracket notation.

Let $n=2 k+1$ be an odd integer. Let $y \in A, x, z, w \in A^{* *}$ and $\left\{x_{\alpha}\right\},\left\{z_{\beta}\right\}$ and $\left\{w_{\gamma}\right\}$ be bounded nets in $A, w^{*}$-converging to $x, z$ and $w$, respectively. Let $t \in B^{(2 k+2)}$ and $\left\{t_{\theta}\right\}$ be a bounded net in $B^{(2 k)}, w^{*}$-converging to $t$. Rewriting the identity (11) in the notation of the expression (8), we have the following identity

$$
\begin{aligned}
\left\langle\pi^{[k] 2}\left(x_{\alpha}, T\left(y z_{\beta}\right), w_{\gamma}\right), t_{\theta}\right\rangle= & \left\langle\pi^{[k] 2}\left(x_{\alpha}, T(y), z_{\beta}^{*} w_{\gamma}\right), t_{\theta}\right\rangle+\left\langle\pi^{[k] 2}\left(x_{\alpha} y^{*}, T\left(z_{\beta}\right), w_{\gamma}\right), t_{\theta}\right\rangle \\
& -\left\langle\pi^{[k] 2}\left(x_{\alpha} y^{*}, T(1), z_{\beta}^{*} w_{\gamma}\right), t_{\theta}\right\rangle
\end{aligned}
$$

for every $x_{\alpha}, z_{\beta}, w_{\gamma}$ and $t_{\theta}$. Hence, by definition of $\pi^{[k] 2}$, we obtain

$$
\begin{aligned}
\left\langle\pi^{[k]}\left(x_{\alpha}, t_{\theta}, w_{\gamma}\right), T\left(y z_{\beta}\right)\right\rangle= & \left\langle\pi^{[k]}\left(x_{\alpha}, t_{\theta}, z_{\beta}^{*} w_{\gamma}\right), T(y)\right\rangle+\left\langle\pi^{[k]}\left(x_{\alpha} y^{*}, t_{\theta}, w_{\gamma}\right), T\left(z_{\beta}\right)\right\rangle \\
& -\left\langle\pi^{[k]}\left(x_{\alpha} y^{*}, t_{\theta}, z_{\beta}^{*} w_{\gamma}\right), T(1)\right\rangle .
\end{aligned}
$$

Since the nets $\left\{z_{\beta}^{*} w_{\gamma}\right\}$ and $\left\{x_{\alpha} y^{*}\right\}$ are $w^{*}$-convergent to $z_{\beta}^{*} w$ and $x y^{*}$, respectively, Proposition 2.1 implies that by taking limits firstly in $\gamma$, then in $\theta$ and after that in $\alpha$, we obtain

$$
\begin{aligned}
\left\langle\pi^{[k+2]}(x, t, w), T\left(y z_{\beta}\right)\right\rangle= & \left\langle\pi^{[k+2]}\left(x, t, z_{\beta}^{*} w\right), T(y)\right\rangle+\left\langle\pi^{[k+2]}\left(x y^{*}, t, w\right), T\left(z_{\beta}\right)\right\rangle \\
& -\left\langle\pi^{[k+2]}\left(x y^{*}, t, z_{\beta}^{*} w\right), T(1)\right\rangle .
\end{aligned}
$$

Since $\left\{y z_{\beta}\right\}$ is $w^{*}$-convergent to $y z$ and $T^{* *}$ is $w^{*}-w^{*}$-continuous, we find that $\left\{T\left(y z_{\beta}\right)\right\}$ is $w^{*}$-convergent to $T^{* *}(y z)$. Hence

$$
\lim _{\beta}\left\langle\pi^{[k+2]}(x, t, w), T\left(y z_{\beta}\right)\right\rangle=\left\langle T^{* *}(y z), \pi^{[k+2]}(x, t, w)\right\rangle .
$$


Also $\left\{T\left(z_{\beta}\right)\right\}$ is $w^{*}$-convergent to $T^{* *}(z)$. Therefore

$$
\lim _{\beta}\left\langle\pi^{[k+2]}\left(x y^{*}, t, w\right), T\left(z_{\beta}\right)\right\rangle=\left\langle T^{* *}(z), \pi^{[k+2]}\left(x y^{*}, t, w\right)\right\rangle .
$$

Since the product and involution of the $\mathrm{C}^{*}$-algebra $A^{* *}$ is weak* continuous, we see that the net $\left\{z_{\beta}^{*} w\right\}$ is $w^{*}$-convergent to $z^{*} w$. Therefore

$$
\begin{aligned}
\lim _{\beta}\left\langle\pi^{[k+2]}\left(x, t, z_{\beta}^{*} w\right), T(y)\right\rangle & =\lim _{\beta}\left\langle\pi^{[k] * \# \# *}\left(z_{\beta}^{*} w, t, x\right), T(y)\right\rangle=\lim _{\beta}\left\langle z_{\beta}^{*} w, \pi^{[k] * \sharp \sharp}(t, x, T(y))\right\rangle \\
& =\left\langle z^{*} w, \pi^{[k] * \# \#}(t, x, T(y))\right\rangle=\left\langle\pi^{[k] * \# *}\left(z^{*} w, t, x\right), T(y)\right\rangle \\
& =\left\langle\pi^{[k+2]}\left(x, t, z^{*} w\right), T(y)\right\rangle .
\end{aligned}
$$

In a similar way, we obtain

$$
\lim _{\beta}\left\langle\pi^{[k+2]}\left(x y^{*}, t, z_{\beta}^{*} w\right), T(1)\right\rangle=\left\langle\pi^{[k+2]}\left(x y^{*}, t, z^{*} w\right), T(1)\right\rangle .
$$

Taking limits on $\beta$ in identity (14) and applying the identities, (15), (16), (17) and (18), we prove that

$$
\begin{aligned}
\left\langle T^{* *}(y z), \pi^{[k+2]}(x, t, w)\right\rangle= & \left\langle T(y), \pi^{[k+2]}\left(x, t, z^{*} w\right)\right\rangle+\left\langle T^{* *}(z), \pi^{[k+2]}\left(x y^{*}, t, w\right)\right\rangle \\
& -\left\langle T(1), \pi^{[k+2]}\left(x y^{*}, t, z^{*} w\right)\right\rangle,
\end{aligned}
$$

or equivalently

$$
\begin{aligned}
\left\langle\pi^{[k+2] 2}\left(x, T^{* *}(y z), w\right), t\right\rangle= & \left\langle\pi^{[k+2] 2}\left(x, T(y), z^{*} w\right), t\right\rangle+\left\langle\pi^{[k+2] 2}\left(x y^{*}, T^{* *}(z), w\right), t\right\rangle \\
& -\left\langle\pi^{[k+2] 2}\left(x y^{*}, T(1), z^{*} w\right), t\right\rangle .
\end{aligned}
$$

Since $t \in B^{(2 k+2)}$ is arbitrary, the desired identity is proved in the odd cases of $n$.

Proposition 3.5. Let $B$ be a unital $C^{*}$-algebra and $A$ be a commutative closed subalgebra of $B$ containing the identity of $B$. Let $T: A \rightarrow B^{(n)}$ be a bounded local triple derivation. Then for each $a, b, c \in A$ with $a^{*} b=b^{*} c=0$ we have $a T(b)^{*} c=0$ and $a T\left(b^{*}\right)^{*} c=0$.

Proof. Let $K$ be a suitable compact Hausdorff space such that $A \simeq C(K)$, the space of all continuous functions on $K$. By Theorem 14 in [9, Chapter 8] $A^{* *} \simeq L^{\infty}(K)$, the space of all bounded, Borel-measurable functions on $K$. Let $a, b, c \in A$ with $a^{*} b=b^{*} c=0$ and set $p=$ $\chi_{S(b)} \in A^{* *}$, where $S(b)=\{t \in K: b(t) \neq 0\}$. It is easy to see that $a p=0, c p=0$ and $b p=b$. Identity (13), combined with Proposition 2.4, implies that

$$
\begin{aligned}
(1-p) T(b)^{*}(1-p)= & {[1-p, T(b), 1-p]=[1-p, T(b p), 1-p]=[1-p, T(b), p(1-p)] } \\
& +\left[(1-p) b^{*}, T^{* *}(p), 1-p\right]-\left[(1-p) b^{*}, T(1), p(1-p)\right]=0 .
\end{aligned}
$$


Therefore, $a T(b)^{*} c=a(1-p) T(b)^{*}(1-p) c=0$, which proves the first identity.

The second identity can be obtained in the same way by considering that by commutativity, $a^{*} b=b^{*} c=0$ implies $a b=b c=0$.

In the following proposition by the notation $T \circ *$, we mean the composition $(T \circ *)(a)=$ $T\left(a^{*}\right)$.

Proposition 3.6. Let $A$ be a commutative subalgebra of a unital $C^{*}$-algebra $B$ which contains the identity of $B$ and $T: A \rightarrow B^{(n)}$ be a bounded local triple derivation with $T(1)=0$. If $n$ is even then $T$ is a derivation and if $n$ is odd then $T \circ *$ is a derivation.

Proof. Let $n$ be even and consider the mapping $G(x)=T\left(x^{*}\right)^{*}$. Proposition 3.5 assures that $a G(b) c=0$, for every $a^{*} b=b^{*} c=0$ in $A$. Now Corollary 2.9 in [10] implies that the mapping $G$ is a generalised derivation, and thus,

$$
\begin{aligned}
T(a b) & =G\left(b^{*} a^{*}\right)^{*}=\left(G\left(b^{*}\right) a^{*}+b^{*} G\left(a^{*}\right)-b^{*} G(1) a^{*}\right)^{*} \\
& =a G\left(b^{*}\right)^{*}+G\left(a^{*}\right)^{*} b-a G(1)^{*} b=a T(b)+T(a) b-a T(1) b=a T(b)+T(a) b,
\end{aligned}
$$

which shows that $T$ is a derivation.

Let $n$ be odd and consider the linear mapping $G(x)=T(x)^{*}$. Proposition 3.5 assures that $a G(b) c=0$, for every $a^{*} b=b^{*} c=0$ in A. Again Corollary 2.9 in [10] implies that the mapping $G$ is a generalised derivation, and thus,

$$
\begin{aligned}
(T \circ *)(a b) & =T\left(b^{*} a^{*}\right)=G\left(b^{*} a^{*}\right)^{*}=\left(G\left(b^{*}\right) a^{*}+b^{*} G\left(a^{*}\right)-b^{*} G(1) a^{*}\right)^{*} \\
& =a G\left(b^{*}\right)^{*}+G\left(a^{*}\right)^{*} b-a G(1)^{*} b=a T\left(b^{*}\right)+T\left(a^{*}\right) b-a T(1) b \\
& =a(T \circ *)(b)+(T \circ *)(a) b,
\end{aligned}
$$

which shows that $T \circ *$ is a derivation.

Proposition 3.7. Let $B$ be a unital $C^{*}$-algebra, and let $T: B \rightarrow B^{(n)}$ be a bounded local triple derivation with $T(1)=0$. Then $T\left(a^{*}\right)=T(a)^{*}$, for every $a \in B$.

Proof. If $n$ be even, proof of [2, Lemma 9] can be restated in this general form to prove the statement. If $n$ be odd the same argument also works except for some changes in involutions. However, for the sake of completeness, we present the proof for odd integers. Let $n$ be an odd integer. Let $a$ be a self-adjoint element in $B$ and $A$ denote the closed subalgebra of $B$ generated by $a$ and the unit of $B$, which is commutative. Since $\left.T\right|_{A}: A \rightarrow B^{(n)}$ is a bounded local triple derivation with $T(1)=0$, Proposition 3.6 implies that $\left.(T \circ *)\right|_{A}=\left.T\right|_{A} \circ *$ is a derivation. Therefore for a unitary element $u \in A$, we have

$$
(T \circ *)\left(u^{*} u\right)=(T \circ *)\left(u^{*}\right) u+u^{*}(T \circ *)(u)=T(u) u+u^{*} T\left(u^{*}\right) .
$$


Since $(T \circ *)\left(u^{*} u\right)=T\left(u^{*} u\right)=T(1)=0$, we obtain

$$
T(u)=-u^{*} T\left(u^{*}\right) u^{*} .
$$

On the other hand $T$ is a local triple derivation and therefore, there exists a triple derivation $D_{u}$ such that $T(u)=D_{u}(u)$. Consequently

$$
\begin{aligned}
T(u) & =D_{u}(u)=D_{u}\left(u u^{*} u\right)=D_{u}[u, u, u]=\left[D_{u}(u), u, u\right]+\left[u, D_{u}(u), u\right]+\left[u, u, D_{u}(u)\right] \\
& =[T(u), u, u]+[u, T(u), u]+[u, u, T(u)] .
\end{aligned}
$$

Now, Proposition 2.4, implies that

$$
T(u)=T(u)+u^{*} T(u)^{*} u^{*}+T(u),
$$

which gives

$$
T(u)=-u^{*} T(u)^{*} u^{*}
$$

Combining equations (19) and (20), we obtain $u^{*} T\left(u^{*}\right) u^{*}=u^{*} T(u)^{*} u^{*}$, which proves that

$$
T\left(u^{*}\right)=T(u)^{*} .
$$

Since $A$ is the linear span of its unitary elements we conclude that $T\left(b^{*}\right)=T(b)^{*}$, for every $b$ in $A$. The arbitrariness of the hermitian element $a$ implies that $T(b)^{*}=T(b)$, for every $b \in B_{s a}$, which combined with the linearity of $T$ proves the desired result.

Lemma 3.8. Let $A$ be a commutative subalgebra of a unital $C^{*}$-algebra $B$ which contains the identity of $B$ and $T: A \rightarrow B^{(n)}$ be a local triple derivation. Then $T(1)^{*}=-T(1)$.

Proof. By definition at the point 1 , there exists a triple derivation $D_{1}: A \rightarrow B^{(n)}$ satisfying $T(1)=D_{1}(1)$. Either $n$ be even or odd, we obtain

$$
\begin{aligned}
T(1) & =D_{1}[1,1,1]=\left[D_{1}(1), 1,1\right]+\left[1, D_{1}(1), 1\right]+\left[1,1, D_{1}(1)\right] \\
& =D_{1}(1)+D_{1}(1)^{*}+D_{1}(1)=T(1)+T(1)^{*}+T(1),
\end{aligned}
$$

which implies the desired result.

Theorem 3.9. Let $B$ be a unital $C^{*}$-algebra. Every bounded local triple derivation $T: B \rightarrow B^{(n)}$ is a triple derivation. 
Proof. Let $T: B \rightarrow B^{(n)}$ be a bounded local triple derivation and set $\widetilde{T}=T-\delta\left(\frac{1}{2} T(1), 1\right)$. Since $\delta\left(\frac{1}{2} T(1), 1\right)$ is a bounded triple derivation we see that $\widetilde{T}$ is also a bounded local triple derivation and either $n$ is even or odd, by Proposition 2.4 and Lemma 3.8, we obtain

$$
\begin{aligned}
\widetilde{T}(1) & =T(1)-\delta\left(\frac{1}{2} T(1), 1\right)(1)=T(1)-\left(\left[\frac{1}{2} T(1), 1,1\right]-\left[1, \frac{1}{2} T(1), 1\right]\right) \\
& =T(1)-\frac{1}{2} T(1)+\frac{1}{2} T(1)^{*}=T(1)-\frac{1}{2} T(1)-\frac{1}{2} T(1)=0 .
\end{aligned}
$$

Let $a$ be a self-adjoint element in $B$ and $A$ be the $C^{*}$-subalgebra of $B$ generated by $a$ and the identity of $B$. Since $A$ is commutative and $\left.\widetilde{T}\right|_{A}$ is a bounded local triple derivation with $\widetilde{T}(1)=0$, Proposition 3.6 implies that $\left.\widetilde{T}\right|_{A}$ is a derivation, whenever $n$ is even and $\left.(\widetilde{T} \circ *)\right|_{A}=$ $\left.\widetilde{T}\right|_{A} \circ *$ is a derivation, whenever $n$ is odd. Thus, we obtain

$$
\widetilde{T}\left(a^{2}\right)=\widetilde{T}(a) a+a \widetilde{T}(a)
$$

either $n$ is even or odd. For every self-adjoint elements $a, b \in B$, we apply (21) to deduce

$$
\widetilde{T}\left((a+b)^{2}\right)=\widetilde{T}(a+b)(a+b)+(a+b) \widetilde{T}((a+b)) .
$$

Now, combining identities (21) and (22), we see that

$$
\widetilde{T}(a \circ b)=\widetilde{T}(a) \circ b+a \circ \widetilde{T}(b)
$$

for each $a, b \in B_{s a}$.

From this point on, let us consider the two different case of even and odd for the integer $n$, separately. If $n$ is even the same argument as in the proof of [2, Theorem 10] can be applied here to establish that $\widetilde{T}$ is a triple derivation. Let $n$ be odd. Linearity of $\widetilde{T} \circ *$ combined with identity (23), establish the equality

$$
(\widetilde{T} \circ *)(a \circ b)=(\widetilde{T} \circ *)(a) \circ b+a \circ(\widetilde{T} \circ *)(b)
$$

for every $a, b \in B$, which proves that $\widetilde{T} \circ *$ is a Jordan derivation. Theorem 6.3 in [6] shows that $\widetilde{T} \circ *$ is an associative derivation. Considering this and applying Proposition 3.7, we see that

$$
\begin{aligned}
\widetilde{T}[a, b, c]= & \frac{1}{2}(\widetilde{T} \circ *)\left(c^{*} b a^{*}+a^{*} b c^{*}\right)=\frac{1}{2}\left((\widetilde{T} \circ *)\left(c^{*}\right) b a^{*}+c^{*}(\widetilde{T} \circ *)(b) a^{*}+c^{*} b(\widetilde{T} \circ *)\left(a^{*}\right)\right. \\
& \left.+(\widetilde{T} \circ *)\left(a^{*}\right) b c^{*}+a^{*}(\widetilde{T} \circ *)(b) c^{*}+a^{*} b(\widetilde{T} \circ *)\left(c^{*}\right)\right) \\
= & \frac{1}{2}\left(\widetilde{T}(c) b a^{*}+c^{*} \widetilde{T}(b)^{*} a^{*}+c^{*} b \widetilde{T}(a)+\widetilde{T}(a) b c^{*}+a^{*} \widetilde{T}(b)^{*} c^{*}+a^{*} b \widetilde{T}(c)\right) \\
= & {[\widetilde{T}(a), b, c]+[a, \widetilde{T}(b), c]+[a, b, \widetilde{T}(c)], }
\end{aligned}
$$

which shows that $\widetilde{T}$ is a triple derivation. 
Finally, since $T=\widetilde{T}+\delta\left(\frac{1}{2} T(1), 1\right)$ is the sum of two triple derivations, we conclude that $T$ is a triple derivation.

\section{Acknowledgements}

The authors would like to thank the anonymous referee for the careful review and the helpful suggestions.

\section{References}

[1] T. J. Barton and R. M. Timoney, Weak* -continuity of Jordan triple products and its applications, Math. Scand., 59 (1986), 177-191.

[2] M. Burgos, F. J. Fernández-Polo, J. J. Garcés and A. M. Peralta, Local triple derivations on $C^{*}$-algebras, Communications in Algebra, 42 (2014), 1276-1286.

[3] M. Burgos, F. J. Fernández-Polo and A. M. Peralta, Local triple derivations on $C^{*}$-algebras and JB* ${ }^{*}$-triples, Bull. London Math. Soc., 46 (2014), 709-724.

[4] S. Dineen, The second dual of a JB*-triple system, Complex Analysis, Functional Analysis and Approximation Theory (Ed. J. Mujica), North-Holland, Amsterdam, 1986.

[5] S. Goldstein, Stationarity of operator algebras, J. Funct. Anal. 118 (1993), 275-308.

[6] B. E. Johnson, Symmetric amenability and the nonexistence of Lie and Jordan derivations, Math. Proc. Cambridge Philos. Soc., 120 (1996), 455-473.

[7] R. V. Kadison, Local derivations, J. Algebra, 130 (1990), 494-509.

[8] A. A. Khosravi and H. R. Ebrahimi Vishki, Regularity of trilinear maps and its application on JB* -triples, to appear 2018.

[9] P. D. Lax, Functional Analysis, John Wiley \& Sons, New York, 2002.

[10] J. Li and Zh. Pan, Annihilator-preserving maps, multipliers, and derivations, Linear Algebra Appl., 423 (2010), 5-13.

[11] M. Mackey, Local derivations on Jordan triples, Bull. London Math. Soc., 45 (2013), 811-824.

[12] J. Martínez and A. M. Peralta, Separate weak* ${ }^{*}$-continuity of the triple product in dual real JB* -triples, Math. Z., 234 (2000), 635-646.

[13] M. Niazi, M. R. Miri and H. R. E. Vishki, Ternary weak amenability of the bidual of a JB*-triple, Banach J. Math. Anal., 11 (2017), 676-697.

[14] A. M. Peralta and B. Russo, Automatic continuity of derivations on $C^{*}$-algebras and JB*-triples, Journal of Algebra, 399 (2014), 960-977.

[15] B. Russo, Structure of JB* -triples, In: Jordan Algebras, Proceedings of the Oberwolfach Conference 1992, Eds: W. Kaup, K. McCrimmon, H. Petersson, de Gruyter, Berlin (1994), 209-280.

Department of Mathematics, University of Birjand, Birjand, Iran.

E-mail: niazi@birjand.ac.ir

Department of Mathematics, University of Birjand, Birjand, Iran.

E-mail: mrmiri@birjand.ac.ir 